halt belasten. Dies hätte den Vorteil, dass der Bundestag in den politischen Haftungsverband integriert wäre und seine Verantwortung nicht mehr auf „die“ in Brüssel abschieben könnte. Vor allem aber würde die Bundesregierung ihren Verpflichtungen aus Art. 23 Abs. 2 S. 2 GG eigennützig nachkommen, denn das Parlament würde immer erst dann entscheiden, wenn es sich ausreichend informiert fühlt.

Eine der ersten Reaktionen der Vertreter der Bundesregierung am 30. Juni 2009 in Karlsruhe war, dass der „Vertrag“ die Hürde genommen habe und sich die Entscheidung in der Praxis kaum auswirke. Die Aufgabe des Bundestags in der kommenden Wahlperiode wird es sein, dieser Feststellung den Boden zu entziehen und dennoch die Handlungsfähigkeit der Europäischen Union vernünftig zu erhalten.

\title{
Neue (alte) Konfliktlinien in der Europapolitik: Die Parlamentsdebatte zum Vertrag von Lissabon im Deutschen Bundestag
}

\author{
Andreas Wimmel
}

In der Parlamentssitzung vom 24. April 2008 hat der Deutsche Bundestag den Gesetzentwurf zur Ratifikation des EU-Reformvertrages von Lissabon, der die in französischen und niederländischen Referenden gescheiterte „Europäische Verfassung“ ersetzen soll, mit der überwältigenden Mehrheit von 515 Stimmen angenommen. ${ }^{1}$ Die Fraktionen von SPD, FDP und Bündnis 90/Die Grünen sowie eine deutliche Mehrheit der CDU/CSU-Abgeordneten haben, wie allgemein erwartet worden war, dem komplizierten Vertragswerk zugestimmt, während die gesamte Linkspartei sowie sieben CDU/CSU-Vertreter, die ihre Ablehnung in Erklärungen teilweise persönlich begründeten, dagegen votierten. ${ }^{2}$ Damit steht diese Ratifikation in der Tradition einer verlässlichen deutschen Europapolitik: Schon die EU-Verträge von Maastricht (1992) und Nizza (2001) waren von allen Bundestagsfraktionen mit Ausnahme der PDS/Linke Liste mit deutlichen Mehrheiten und ohne große zwischenparteiliche Konflikte ratifiziert worden. ${ }^{3}$ Der breite Konsens der beiden Volkspar-

1 Nach dem erfolgreichen zweiten Referendum in Irland und der Unterzeichnung durch den tschechischen Präsidenten Vaclav Klaus ersetzt seit dem 1. Dezember 2009 der Vertrag von Lissabon die geltenden EU-Grundlagenverträge. Dieser lange Ratifizierungsprozess ändert jedoch prinzipiell nichts an der Positionierung deutscher Parteien zur Europapolitik im Rahmen der Bundestagsdebatte zum Vertrag von Lissabon, die in diesem Beitrag vergleichend dokumentiert wird.

2 Das Endergebnis der namentlichen Abstimmung lautet: 574 abgegebene Stimmen, davon 515 ja, 58 nein, eine Enthaltung. Gegen den Gesetzentwurf stimmten alle 49 Abgeordneten der Partei Die Linke, die sieben CDU/CSU-Abgeordneten Alexander Dobrindt, Herbert Frankenhauser, Peter Gauweiler, Paul Lehrieder, Georg Nüßlein, Marion Seib und Willy Wimmer (Neuss) sowie die beiden fraktionslosen Abgeordneten Henry Nitzsche und Gert Winkelmeier; der Abgeordnete Anton Hofreiter (Bündnis 90/Die Grünen) enthielt sich.

3 Abstimmungsergebnis Nizza: 605 abgegebene Stimmen, davon 571 Ja-Stimmen (alle Abgeordneten von CDU/CSU, SPD und Bündnis 90/Die Grünen), 32 Nein-Stimmen (alle PDS-Abgeord- 
teien in europapolitischen Grundsatzfragen, der seit jeher durch die Idee einer wirtschaftlich und politisch integrierten Staatengemeinschaft geprägt gewesen ist, bestand auch in der Großen Koalition, die in vielen anderen Politikbereichen keine Einigkeit erzielen konnte, fort.

Gibt es dennoch Anzeichen für Spannungen dieses parteiübergreifenden deutschen Europakonsenses? Diese Kurzdokumentation der Parlamentsdebatte zum EU-Vertrag von Lissabon, die der namentlichen Abstimmung vorausgegangen war, bestätigt einerseits die Kontinuität europapolitischer Leitlinien im deutschen Parteiensystem. ${ }^{4}$ Nach wie vor besteht kein Zweifel an der pro-europäischen Grundhaltung aller Fraktionen außer der Linkspartei, die zwar die Idee europäischer Integration nicht prinzipiell ablehnt, aber eine radikale Neuausrichtung europapolitischer Ziele und Inhalte fordert. Andererseits offenbart eine detaillierte Inhaltsanalyse der Redebeiträge der CDU/CSU- und der SPD-Vertreter im Vergleich mit den Bundestagsdebatten zu den Verträgen von Maastricht und Nizza die Wiederbelebung einer alten Konfliktlinie in der deutschen Europapolitik. Während der überwiegende Teil der CDU/CSU-Fraktion an seiner pro-europäischen Rhetorik festhält, wurde insbesondere von SPD-Spitzenpolitikern die soziale Frage wieder verstärkt im EU-Kontext problematisiert und das Zukunftsideal eines „sozialen Europas“ ausgerufen, das durch EURegulierungen (zum Beispiel Unternehmenssteuern, Regulierungen des Arbeitsmarkts, Arbeitnehmerrechte) die freie Marktwirtschaft zugunsten des Gemeinwohls zähmen soll. Diese unterschiedlichen Auffassungen zur sozialen Dimension des Integrationsprozesses, die von der neuen Linkspartei wieder auf die politische Tagesordnung gesetzt wurde, könnten sich zu einer echten Kontroverse entwickeln, die das deutsche Parteiensystem in zwei Europalager zu spalten droht.

\section{Die Bundestagsdebatte zum EU-Reformvertrag von Lissabon im Vergleich}

In den folgenden Abschnitten werden die zentralen Positionen und Argumente der beiden Hauptredner der Bundestagsfraktionen von CDU/CSU und SPD zum EU-Reformvertrag von Lissabon sowie Auszüge aus persönlichen Erklärungen zu den namentlichen Abstimmungen dokumentiert und mit den Bundestagsdebatten zu der Ratifikation der Verträge von Maastricht (1992) und Nizza (2001) verglichen. ${ }^{5}$ Damit soll überprüft werden, ob und inwieweit die Grundhaltungen und Zukunftsideen der beiden Volksparteien zur europäischen Integration über die Jahre konstant geblieben sind oder sich zumindest tendenziell

nete) und zwei Enthaltungen (1 FDP, 1 PDS). Maastricht: 567 abgegebene Stimmen, davon 543 mit Ja, 16 mit Nein (alle Abgeordneten der PDS/Linke Liste, zwei SPD-Abgeordnete und vier Grünen-Vertreter) und acht Enthaltungen (2 CDU/CSU, 6 SPD).

4 Siehe Markus Jachtenfuchs, Die Konstruktion Europas. Verfassungsideen und institutionelle Entwicklung, Baden-Baden 2002; Gisela Müller-Brandeck-Bocquet (Hrsg.), Deutsche Europapolitik von Konrad Adenauer bis Gerhard Schröder, Opladen 2002; Heinrich Schneider / Mathias Jopp I Uwe Schmalz (Hrsg.), Eine neue deutsche Europapolitik? Rahmenbedingungen - Problemfelder - Optionen, Bonn 2001.

5 Die „Hauptredner“ sind jeweils die beiden Parteienvertreter mit den ranghöchsten politischen Ämtern und/oder den längsten Redezeiten. Die Beiträge der weiteren Redner jeder Partei werden nur dann explizit erwähnt, wenn sich ihre Positionen wesentlich von denjenigen der Hauptredner unterscheiden. 
gewandelt haben. Im Mittelpunkt steht die vor allem von der SPD-Fraktion in dieser Schärfe neu eingeführte Forderung nach einem „sozialen Europa“ sowie die verfassungsrechtlich begründete Kritik am Vertrag von Lissabon von einigen bayrischen CSU-Bundestagsabgeordneten, die unter anderem den Verlust nationaler Souveränitätsrechte und demokratischer Legitimation beklagen.

Die Konzentration auf die beiden großen Volksparteien ${ }^{6}$ begründet sich mit deren potentieller Vetoposition, sowohl auf nationaler als auch auf europäischer Ebene: Die Entstehung einer neuen Konfliktlinie zwischen diesen beiden Parteien wäre für die deutsche Europapolitik und damit für die Zukunft der Europäischen Union besonders folgenreich, da die Ratifikation von EU-Verträgen mit Zweidrittelmehrheit erfolgen muss und somit, im Gegensatz zu den „kleineren“ Parteien, eine der beiden Volksparteien EU-Vertragsratifikationen allein blockieren könnte. Zudem bestünde vor allem in Zeiten Großer Koalitionen die Gefahr, dass sich die deutschen Vertreter in den EU-Institutionen, vor allem im Ministerrat, aus Rücksichtnahme auf den Regierungspartner bei wichtigen Zukunftsentscheidungen enthalten müssten, wenn zwischen den beiden Parteien keine gemeinsamen Positionen gefunden werden können.

Die Parlamentsdebatten werden entlang drei potentieller Konfliktfelder miteinander verglichen, um die Unterschiede und Ähnlichkeiten der Europapolitik der Parteien erfassen zu können. Erstens, die grundsätzlichen Einstellungen der beiden Parteien zum europäischen Integrationsprojekt sowie deren Zukunftsideen zur Finalität der EU: Wie bewerten sie die bisherige deutsche Europapolitik insgesamt, und welchen institutionellen Endzustand streben sie für die EU an? Zweitens, die spezifische Bewertung des jeweils im Anschluss an die Debatte zu ratifizierenden EU-Vertrages: Wie wiegen die Parteien die Vor- und Nachteile der mit dem Vertrag verbundenen institutionellen und politischen Reformen ab, und welche Versäumnisse oder Mängel kritisieren sie? Und schließlich zwischenparteiliche oder innerparteiliche Meinungsverschiedenheiten oder offene Konflikte in europapolitischen Fragen: Haben die Redner derselben Parteien unterschiedliche Einstellungen vertreten beziehungsweise wurde innerparteilich Kritik geübt, und wurde die Europapolitik anderer Parteien kritisiert beziehungsweise für Positionen argumentiert, die mit den Vorstellungen der anderen Parteien nicht zu vereinbaren sind? Der Vergleich umfasst also zwei Dimensionen, nämlich (1) die Positionen der Parteien, zum Beispiel der SPD, zu den EU-Verträgen von Maastricht, Nizza und Lissabon im Zeitvergleich, und (2) die Positionen von CDU/ CSU und SPD zu einem bestimmten EU-Vertrag, beispielsweise zum Reformvertrag von Lissabon, zum selben Zeitpunkt.

Die Inhaltsanalyse von Parlamentsdebatten anlässlich der Ratifikation von EU-Verträgen stellt eine etablierte Methode dar, um Parteipositionen zu europapolitischen Angelegenheiten zu erfassen. Aufgrund der großen Bedeutung des Themas treten in der Regel die jeweiligen Spitzenpolitiker an das Rednerpult und geben die Leitlinien der Partei vor, während

6 Inwieweit CDU und SPD trotz ihrer dramatisch rückläufigen Mitgliederzahlen und schlechter Wahlergebnisse in einigen Bundesländern noch als echte „Volksparteien“ bezeichnet werden können, wird seit einiger Zeit auch in den Medien lebhaft diskutiert; vgl. Christoph Gunkel / Roland Nelles / René Pfister, Volksparteien ohne Volk, in: Der Spiegel vom 7. Juli 2008, S. 40 - 42; Bernd Ulrich, Niedergang der Volksparteien - Gefahr für die Demokratie?, in: Die Zeit vom 9. Oktober 2008, S. 3. Da sich dieser Begriff jedoch als Schlagwort zur Bezeichnung dieser beiden Parteien durchgesetzt hat, wird in diesem Beitrag daran festgehalten. 
der Europapolitik in Partei- und Wahlprogrammen traditionell verhältnismäßig wenig Raum gegeben wird. ${ }^{7}$

\section{Christlich-Demokratische Union/Christlich-Soziale Union (CDU/CSU)}

Die beiden Hauptredner der CDU/CSU-Fraktion in der Bundestagsdebatte zum Vertrag von Lissabon waren Bundeskanzlerin Angela Merkel und der frühere bayrische Ministerpräsident Günther Beckstein. Merkel eröffnete die Aussprache, indem sie kurz den europäischen Integrationsprozess insgesamt als Erfolgsgeschichte würdigte und vor allem die grundlegende Einigkeit im Bundestag in europapolitischen Angelegenheiten „nahezu über alle Fraktionsgrenzen hinweg“ herausstrich (Merkel, 16451 D). Den Vertrag von Lissabon im Speziellen bezeichnete sie als eine „neue Grundlage für Europa, (...) die solide und von Bestand ist" (Merkel, 16451 D), weil er im Gegensatz zu vorigen EU-Verträgen kein Verfallsdatum oder eine Revisionsklausel beinhalte und grundlegende Änderungen in nächster Zukunft nicht geplant seien. Im Einzelnen verwies die Kanzlerin auf eine ganze Reihe von „erheblichen Vorteilen“ (Merkel, 16452 B), die aus dem neuen Vertrag für Deutschland und für die EU insgesamt resultierten: Erstens sichere er die Entscheidungs- und Handlungsfähigkeit, da der Rat nun „überwiegend mit Mehrheit statt mit Einstimmigkeit“ beschließe und so „Stillstand und Blockaden sehr viel besser überwunden“ werden könnten (Merkel, 16452 C). Darüber hinaus führe er unter anderem zweitens zu einer „gerechteren Gewichtung der Stimmen “, da bei Ratsentscheidungen die Bevölkerungsgröße nun mehr ins Gewicht falle. Drittens bringe der Reformvertrag eine klarere „Kompetenzordnung“, die die Zuständigkeiten der Mitgliedstaaten besser festlege. Viertens erleichtere er die Zusammenarbeit „in einem ganz wichtigen Feld der Politik, nämlich in der Innen- und Justizpolitik“. Und fünftens würden damit die Grundlagen für einen „gemeinsamen Klimaschutz und für eine solidarische Zusammenarbeit im Energiebereich" geschaffen (Merkel, 16452 D). Ferner unterstützte Merkel alle wichtigen institutionellen Neuerungen: die Stärkung des Europäischen Parlaments (EP) und der nationalen Parlamente, die Verkleinerung der Kommission sowie die Einrichtung des Ratspräsidenten und des Hohen Vertreters für Außenpolitik, auch wenn noch nicht alle Kompetenzfragen geklärt seien. Alles in allem jedoch, so Merkel, sei der Vertrag von Lissabon „ein Gewinn für Deutschland“ und könne „in seiner Bedeutung nicht hoch genug eingeschätzt" werden (Merkel, 16453 A). Insofern knüpfte sie mit ihrer Rede nahtlos an die pro-europäische Tradition der Christdemokraten an und betonte, sie hätte sogar eine stärkere Integration in einigen Punkten begrüßt, die teilweise im gescheiterten EU-Verfassungsvertrag vorgesehen waren.

7 Grundlage der Dokumentation sind die offiziellen Plenarprotokolle der Bundestagsdebatten zum Vertrag von Lissabon vom 24. April 2008, http://dip21.bundestag.de/dip21/btp/16/16157.pdf (Abruf am 6. Oktober 2009), zum Vertrag von Nizza vom 18. Oktober 2001, http://dip21.bundestag.de/dip21/btp/14/14195.pdf (Abruf am 6. Oktober 2009) und zum Vertrag von Maastricht vom 2. Dezember 1992, http://dip21.bundestag.de/dip21/btp/12/12126.pdf (Abruf am 6. Oktober 2009), die auch persönliche Erklärungen nach $\$ 31$ GO zu den namentlichen Abstimmungen enthalten. Als Quellenangabe wird immer auf die Seitenzahl dieser Protokolle verwiesen. 
Der zweite Redner, Günther Beckstein (CSU), unterstützte grundsätzlich die Ausführungen der Bundeskanzlerin, brachte aber auch seine Unzufriedenheit mit einigen Elementen des neuen Vertrages und mit dem Status quo der EU allgemein zum Ausdruck, ohne jedoch die sieben Abweichler aus den eigenen Reihen, die in der anschließenden Abstimmung mit Nein votierten (siehe Fußnote 2), zu erwähnen. Nachdem er den Vertrag von Lissabon insgesamt als „Fortschritt für Europa“ gewürdigt hatte, der die Union „ein Stück weit handlungsfähiger und ein Stück weit demokratischer" (Beckstein, 16467 A) mache und die gemeinsame Außen- und Sicherheitspolitik stärke, erinnerte er seine Parteifreunde daran, dass „Europa kein einheitlicher Staat, (...) kein Bundesstaat“ werden dürfe und solle, der die „Staatlichkeit der Bundesrepublik Deutschland beeinträchtigt“, wie dies im Urteil des Bundesverfassungsgerichts zum Maastricht-Vertrag festgestellt worden sei. ${ }^{8}$ Zudem machte er deutlich, dass „nicht jedes Problem in Europa ein Problem für Europa“ sei, das der „Regelungskompetenz der Europäischen Union“ unterliege („Ich sage hierzu: Bayern wird der Wächter der Subsidiarität sein“), unterstützte aber gleichzeitig ein Mehr an Europa „bei der Gestaltung der Globalisierung“ und der „Bändigung der globalen Finanzmärkte“. Es liege im deutschen und im europäischen Interesse, dass „Mindeststandards für Arbeitnehmer nicht nur in Europa, sondern auch in anderen Ländern und Wirtschaftsräumen der Welt eingehalten werden, die mit uns konkurrieren“ (Beckstein, 16468 A). Beckstein bekannte sich zur Fortsetzung des Integrationsprozesses, mahnte aber nachdrücklich an, dass „Subsidiarität und Eigenstaatlichkeit von Bund und Ländern die Grundlage sein“ müssten.

\subsection{Die Verträge von Nizza und Maastricht}

Ein Vergleich mit den Redebeiträgen der CDU/CSU-Delegierten zu den Verträgen von Nizza und Maastricht bestätigt die Stabilität christdemokratischer Europapolitik, verdeutlicht jedoch auch einige Anpassungen: In der Nizza-Debatte präsentierten sich der damalige Vorsitzende der CDU/CSU-Fraktion Friedrich Merz sowie der frühere europapolitische Sprecher Peter Hintze voll und ganz als überzeugte Europäer (Merz: „Wir brauchen in vielen Bereichen mehr Europa und ein Mehr an europäischer Integration") und begrüßten die aus dem Vertragswerk resultierenden Reformen nachdrücklich, vor allem die institutionellen Neuregelungen, die den Weg freimachten für die Osterweiterung der Union. Beide unterstützten die Stärkung des EP und begrüßten, schon mit Blick auf den damals geplanten Verfassungsprozess, dass „,in einem Konvent oder in einer ähnlichen Institution auch die Parlamente der Mitgliedstaaten bei der weiteren Entwicklung der Europäischen Union beteiligt werden" (Merz, 18987 B) sollten. Mehr noch, unter dem Eindruck der Terroranschläge vom 11. September 2001 hätten sich beide weitere Integrationsfortschritte gewünscht, die deutlich über die Vereinbarungen der Regierungskonferenz von Nizza hinausgegangen wären, insbesondere in der gemeinsamen Außen- und Sicherheitspolitik sowie der Innen- und Rechtspolitik. Vor allem Hintze kritisierte die rot-grüne Bundesregierung

8 Vgl. Doris König, Das Urteil des Bundesverfassungsgerichts zum Vertrag von Maastricht. Ein Stolperstein auf dem Weg in die europäische Integration?, in: Zeitschrift für ausländisches öffentliches Recht und Völkerrecht, 54. Jg. (1994), S. 17 - 94; Joachim Wieland, Germany in the European Union: The Maastricht Decision of the Bundesverfassungsgericht, in: European Journal of International Law, 5. Jg. (1994), H. 2, S. $259-266$. 
dafür, dass es ihr nicht gelungen sei, im Rat der europäischen Staats- und Regierungschefs tiefgreifendere Reformvorhaben durchzusetzen, die mehr als den kleinsten gemeinsamen Integrationsnenner darstellten (Hintze, $19001 \mathrm{~B}$ ).

Die Maastricht-Debatte wurde von einer außerordentlich langen und detaillierten Rede von Helmut Kohl dominiert, in der der damalige Bundeskanzler die Erfolgsgeschichte der europäischen Einigung und die Verdienste der Europäischen Gemeinschaft, insbesondere für die Schaffung dauerhaften Friedens, historisch würdigte und argumentierte, es gebe keine Alternative zur Fortsetzung der europäischen Integration („Wir alle in Europa und vor allem die Deutschen brauchen aus ureigenstem Interesse die Einigung Europas"), die der ehemalige Bundesfinanzminister Theodor Waigel punktuell ergänzte. ${ }^{9}$ Beide führten aus, dass die Bundesregierung den Maastricht-Vertrag, der zu einer erheblichen Vertiefung und Weiterentwicklung der europäischen Integration, vor allem im wirtschafts- und währungspolitischen Bereich, aber auch in der Gemeinsamen Außen- und Sicherheitspolitik sowie der Innen- und Rechtspolitik führte, voll und ganz unterstütze und mit dem Vertragswerk „ein ausbaufähiges Fundament für die Fortentwicklung“ (Kohl, 10825 B) gelegt worden sei, vor allem mit vorausschauendem Blick auf das zu der Zeit noch visionäre Ziel der Osterweiterung, die Kohl schon damals angestrebt hatte. Im weiteren Verlauf stellte der Kanzler alle wichtigen Reformen vor, die der Maastricht-Vertrag beinhaltete, und warb nachhaltig für die damit verbundenen Vorteile, zuallererst für die vollständige Wirtschafts- und Währungsunion und die Einrichtung einer unabhängigen Europäischen Zentralbank. Waigel konzentrierte sich ebenfalls auf die letzten Punkte und verteidigte vehement die positiven Effekte einer einheitlichen europäischen Währung, auch gegen die Kritiker in den eigenen Reihen (Waigel, 10840 A).

\subsection{Zwischenfazit: Die Europapolitik von CDU/CSU}

Vergleicht man die Ausführungen der beiden CDU/CSU-Hauptredner der Debatten zu den Verträgen von Nizza und Maastricht, zeigen sich kaum inhaltliche Unterschiede, denn alle vier Parteivertreter verteidigten die pro-europäische Leitlinie und befürworteten eher ein Mehr als ein Weniger an europäischer Integration. Berücksichtigt man jedoch die ausführlichen persönlichen Erklärungen einiger Abgeordneter, zeigt sich, dass der MaastrichtVertrag innerparteilich nicht unumstritten war, obwohl die Fraktion ihn damals (abgesehen von zwei Enthaltungen) noch fast geschlossen unterstützte. Ein Abgeordneter konnte der seiner Ansicht nach voreiligen Währungsunion nicht zustimmen, fünf weitere Parlamentarier argumentierten, dass sie den Vertrag nur mit diversen Vorbehalten (vor allem: Verringerung von Demokratie und Kontrolle durch nationale Parlamente) akzeptieren könnten, was in der Nizza-Debatte überhaupt nicht mehr der Fall war (keine kritischen Nebenredner, keine von der Parteilinie abweichenden persönlichen Erklärungen). Erst in der aktuellen

9 Weitere CDU/CSU-Redner in der langen und intensiv geführten Maastricht-Debatte waren Kurt Falthauser, Thomas Goppel, Karl Lamers, Hartmut Koschyk, Franz Möller, Rupert Scholz und Renate Hellwig, die alle die Kernpositionen der beiden Hauptredner unterstützten oder sich in bestimmten Teilbereichen (GASP, Stärkung des EP) sogar mehr Integration gewünscht hätten. In der Abstimmung stimmten, abgesehen von zwei Enthaltungen (Joachim Clemens, Heinrich Lummers), alle CDU/CSU-Abgeordneten dem Maastricht-Vertrag zu. 
Debatte zum Vertrag von Lissabon ist diese alte innerparteiliche Konfliktlinie, nun vor allem zwischen den Schwesterparteien CDU und CSU, in neuer Intensität aufgebrochen: Sieben CSU-Abgeordnete stimmten mit Nein und begründeten ihre Ablehnungen mit nationalen Souveränitätsverlusten und verfassungsrechtlichen Bedenken, woraufhin MdB Peter Gauweiler (CSU) vor dem Bundesverfassungsgericht Klage gegen den Vertrag einreichte, ebenso wie Abgeordnete der Linkspartei. ${ }^{10}$ Außerdem erkannten die beiden Hauptredner der Fraktion erstmals, natürlich eingebettet in eine pro-europäische Rhetorik, die soziale Dimension als Teil der zukünftigen Europapolitik an und betonten, mit Blick auf die innerparteiliche Kritik, den Wert nationaler Staatlichkeit und parlamentarischer Souveränität in einer Klarheit (Beckstein), die in den Aussprachen zu früheren EU-Verträgen so nicht zum Ausdruck kam.

\section{Sozialdemokratische Partei Deutschlands (SPD)}

Der damalige SPD-Vorsitzende und rheinland-pfälzische Ministerpräsident Kurt Beck sowie die Stellvertretende Vorsitzende der SPD-Fraktion, Angelika Schwall-Düren, waren die beiden Hauptredner in der Debatte zum Vertrag von Lissabon für die Sozialdemokraten. Zu Beginn seiner Rede verwies Beck auf den langen Kampf der deutschen Sozialdemokratie für ein geeintes Europa und würdigte den Integrationsprozess insgesamt als „Erfolgsgeschichte“, der mit dem Lissabon-Vertrag nun seinen „vorläufigen Abschluss“ gefunden habe (Beck, 16456 D). Obwohl er auch die „wirtschaftliche Bedeutung“ der EU hervorhob, den Nutzen des gemeinsamen Binnenmarktes für die deutsche Exportwirtschaft betonte und die Einführung des Euro rückblickend befürwortete, stellte er nachdrücklich fest, dass „für uns an diesem Europa immer noch ein ganz entscheidender Teil, nämlich das, was wir soziales Europa nennen" (Beck, 16458 C), fehle, wofür er vom Fraktionsvorsitzenden der Linkspartei, Oskar Lafontaine, symbolträchtig mit Beifall bedacht wurde. Allerdings, und das bleibt ein wichtiger Unterschied zwischen der SPD und der Linkspartei, betonte Beck, dass der Vertrag von Lissabon seiner Meinung nach ausreichend Optionen beinhalte, um ein soziales Europa zu verwirklichen. Der Vertrag biete die Chance, neben der ökonomischen „die soziale Dimension dieses Europas in den Mittelpunkt unserer Weiterentwicklungsbemühungen zu stellen“ (Beck, 16458 D). Im weiteren Verlauf seiner Rede stellte Beck diverse sozialpolitische Maßnahmen vor, deren Umsetzung seine Fraktion in den nächsten Jahren auf europäischer Ebene unterstützen und vorantreiben wolle, unter anderem eine „Stärkung der Arbeitnehmerrechte im europäischen Binnenmarkt" und, wenn auch nicht explizit so bezeichnet, die Durchsetzung von europaweiten Mindestlöhnen, die den Menschen ermöglichen würden, durch „,eigene Arbeit und Anstrengung für sich und ihre Familien zu sorgen“ (Beck, 16458 D). In diesem Kontext merkte Beck an, dass ihm insbesondere die Rechtsprechung des Europäischen Gerichtshofs Sorgen bereite, die „die ökonomische Be-

10 Vgl. Karl Albrecht Schachtschneider, Verfassungsrechtliche Argumente gegen den Vertrag von Lissabon, in: Leviathan, 36. Jg. (2008), H. 3, S. 317 - 343. Am 30. Juni 2009 hat das Bundesverfassungsgericht den Lissabon-Vertrag in seinem Urteil nur unter Auflagen akzeptiert; vgl. Nikolas Busse, Das Urteil des Bundesverfassungsgerichts über den Lissabon-Vertrag: Fahrpläne mit mehreren Vorbehalten, in: FAZ vom 1. Juli 2009, S. 2. Siehe dazu den Beitrag von Roland Lhotta und Jörn Ketelhut in diesem Heft. 
trachtung absolut in den Vordergrund“ stelle, so dass „der soziale Ausgleich dahinter deutlich zurücktritt“ (Beck, 16459 B). Man wolle keinen Wettbewerb „um die schnellere soziale Abwärtsspirale (...), sondern einen Wettbewerb um die Teilhabe der Bürgerinnen und Bürger von Malta bis Schweden“. Das, so Beck emphatisch, sei „unsere Vorstellung von einem sozial gerechten Europa" im erweiterten Binnenmarkt (Beck, 16459, C).

Die zweite Rednerin für die SPD-Fraktion, die Abgeordnete Angelika Schwall-Düren, unterstützte Becks vorherige Ausführungen, insbesondere befürwortete sie die institutionellen Neuerungen, die der Union mehr demokratische Legitimation verleihen sollen, wie vor allem die Stärkung der nationalen Parlamente und die Möglichkeit, ein Bürgerbegehren einzubringen: „Man kann es nicht genug unterstreichen: Die Demokratie gewinnt“ (Schwall-Düren, 16464 D). Ganz besonders wichtig sei jedoch auch ihr „die Stärkung der sozialen Dimension“ nach der Osterweiterung, für die der Vertrag ihrer Ansicht nach eine gute Grundlage darstelle, denn er „biete den notwendigen Spielraum, um aus der Wirtschaftsunion eine soziale Union zu machen, um die soziale Union der Wirtschaftsunion an die Seite zu stellen“, unter anderem durch „die Einführung von Mindestlöhnen in allen Bereichen“. Sie kritisierte explizit die Linkspartei für deren Behauptung, der Vertrag bedeute die Festlegung auf einen "neoliberalen Finanzmarktkapitalismus und den Verzicht auf Sozialstaatlichkeit“ (Schwall-Düren, 16465 A). Lothar Bisky, der zuvor für die Fraktion der Linkspartei gegen den Vertrag von Lissabon opponiert hatte, warf sie vor, er betreibe „Linksnationalismus und das Schüren von Angst“, was die Bürger davon abhalte, die Chancen dieses Vertrages zu sehen (Schwall-Düren, 16465 B). Wie schon Beck argumentierte auch Schwall-Düren gegenüber der Linkspartei, der Vertrag würde die Ausgangsbedingungen dafür verbessern und nicht verschlechtern: „Europa gelingt gemeinsam, und auch Europa sozial gelingt gemeinsam. Lassen Sie es uns anpacken" (Schwall-Düren, 16465 C).

\subsection{Die Verträge von Nizza und Maastricht}

Vergleicht man die Reden der SPD-Vertreter zum Vertrag von Lissabon nur mit der NizzaDebatte, könnte dieser Schwenk in Richtung eines sozialen Europas fast schon als echter Politikwechsel aufgefasst werden. In den Ausführungen des damaligen Bundeskanzlers Gerhard Schröder und des Abgeordneten Günter Gloser spielte die soziale Dimension keine Rolle, lediglich einmal wurde am Rande recht allgemein gefordert, das „europäische Sozialmodell“ als Antwort auf die Globalisierung weiterzuentwickeln (Gloser, 18990 C), ohne dieses Thema jedoch weiter zu problematisieren. Stattdessen wurde das Projekt der europäischen Integration einmal mehr als „die größte Erfolgsgeschichte des 20. Jahrhunderts“ gewürdigt (Schröder, 18981 C) und, mit den Eindrücken der Terroranschläge in den USA im Hinterkopf, weitere Integrationsschritte in diversen innen- und rechtspolitischen Bereichen (Europol, Terrorismusbekämpfung) befürwortet sowie ein geschlossenes Vorgehen in der Außen- und Sicherheitspolitik gefordert. Der Nizza-Vertrag wurde voll und ganz begrüßt, insbesondere weil er "die notwendigen Voraussetzungen für die Erweiterung“ schaffe, die von der damaligen rot-grünen Bundesregierung maßgeblich vorangetrieben worden sei: „Deutschland ist und bleibt einer der Motoren des Erweiterungsprozesses“ (Schröder, 18984 A). Alle mit dem Vertrag von Nizza verbundenen institutionellen Veränderungen erfuhren von der SPD-Fraktion breite Unterstützung, und mit Blick auf den ebenfalls gewünschten Verfassungskonvent wurde bereits im Jahr 2001 die Notwendigkeit weiterer Reformen für 
„mehr Transparenz, mehr Effizienz und natürlich mehr Bürgernähe“ (Schröder, 18984 B) angemahnt.

Dass die aktuelle EU-Kritik der SPD an einer fehlenden sozialen Dimension kein neues Phänomen ist, zeigt jedoch ein Vergleich mit der Maastricht-Debatte. Der Vertrag von Maastricht war innerhalb der SPD-Fraktion alles andere als unumstritten, nicht zuletzt aufgrund der starken Fokussierung auf die Wirtschafts- und Währungsunion, was in den Beiträgen der beiden Hauptredner für die SPD-Fraktion zum Ausdruck kam: Während Günter Verheugen als überzeugter Europäer versuchte, die Kritik der eigenen Fraktion am Maastricht-Vertrag argumentativ zu entkräften und entschieden für das auf europäischer Ebene mühsam ausgehandelte Gesamtpaket zur Fortsetzung des Integrationsprozesses warb (Verheugen, 10832 B), nahm die damalige europapolitische Sprecherin der Fraktion, Heidemarie Wieczorek-Zeul, diverse Kritikpunkte auf, die in einigen Reden anderer SPDAbgeordneter sowie in persönlichen Erklärungen formuliert wurden. Nachdem sie die Idee einer immer engeren Europäischen Union und den Beitrag Willy Brandts zur europäischen Einheit emphatisch gewürdigt hatte, kritisierte sie, es fehle „dem Maastricht-Vertrag an der Verankerung des Ziels einer Sozialunion und einer Umweltunion“ (Wieczorek-Zeul, 10815 A). Zwei SPD-Abgeordnete stimmten mit Nein, sechs enthielten sich, und zahlreiche weitere Genossen brachten in persönlichen Sammelerklärungen zum Ausdruck, dass sie dem Vertrag nur mit großen Vorbehalten zustimmen könnten, denn ihrer Ansicht nach seien „die Ergebnisse von Maastricht im sozialpolitischen Bereich völlig unbefriedigend“ (Gemeinsame Erklärung, 10900 C) oder würden „im Bereich der Umweltpolitik erhebliche Defizite" (Mehl, 10877 C) aufweisen. ${ }^{11}$

\subsection{Zwischenfazit: Die Europapolitik der SPD}

Rückblickend bleibt festzuhalten, dass die jüngsten Forderungen der SPD nach einer Stärkung der sozialen Dimension keine Neuausrichtung sozialdemokratischer Europapolitik bedeuten. Im Gegenteil, diese skeptische Haltung gegenüber einer häufig als „neoliberal“ gebrandmarkten EU-Wirtschaftspolitik war bereits seit Maastricht im stärker linksorientierten Lager der Sozialdemokraten unterschwellig vorhanden, auch wenn in der Nizza-Debatte keine Außenwirkung erzeugt werden konnte. Das Dilemma der SPD-Europapolitik besteht letztlich darin, dass die Partei zwar die ursprünglichen Kernideen der europäischen Integration stets befürwortet und unterstützt hat, aber sich die praktischen Folgewirkungen des immer weiter deregulierten Binnenmarktes inzwischen nicht mehr mit ihren Vorstellungen von nationaler Sozialpolitik vereinbaren lassen und sie deswegen ein „soziales Europa " fordern muss. Während dieses Spannungsverhältnis in der Maastricht-Debatte von den beiden Hauptrednern nur am Rande problematisiert wurde, obwohl damals die Vorbehalte

11 Gegen den Vertrag stimmten die SPD-Abgeordneten Eike Ebert sowie Peter Conradi, der seine Ablehnung in erster Linie mit mangelhafter demokratischer Legitimation und zu großen nationalen Souveränitätsverlusten begründete; die Abgeordneten Brigitte Adler, Liesel Hartenstein, Albrecht Müller (Pleisweiler), Hermann Scheer, Otto Schily und Hans-Günther Toetemeyer enthielten sich; insgesamt 56 Abgeordnete begründeten teilweise in Sammelerklärungen, warum sie sich enthalten hatten beziehungsweise dass sie dem Vertrag trotz erheblicher Unzulänglichkeiten, meist im sozial- und umweltpolitischen Bereich, zugestimmt hatten, weil sie insgesamt seine Vorteile höher bewerteten als die Nachteile. 
in der Fraktion größer waren als heute, haben die beiden Hauptredner der Lissabon-Debatte unter dem Druck der Linkspartei nun den Anspruch, die soziale Dimension der EU nachhaltig ausbauen zu wollen, als neue Leitlinie der SPD-Europapolitik fest verankert. Durch die Aussage, der Vertrag von Lissabon biete eine solide Grundlage, um den sozialen Ausgleich in Europa zu stärken, hat sich die Partei selbst in eine Zwickmühle manövriert, denn wenn man die neue europapolitische Maxime ernstnimmt, dürfte die SPD kaum noch Regelungen auf EU-Ebene zustimmen, die diesem Ziel nicht dienlich sind. Insofern bleibt abzuwarten, ob die Sozialdemokraten trotz dieses Widerspruchs weiterhin grundlegenden EU-Vertragsänderungen positiv gegenüberstehen oder sich mehr und mehr der grundsätzlich ablehnenden Haltung der Linkspartei annähern werden.

\section{Kontinuität und Wandel in der Europapolitik von CDU/CSU und SPD}

Die Europapolitik der deutschen Bundestagsparteien (mit Ausnahme der PDS/Die Linke) zeichnet sich auch fünfzig Jahre nach den Römischen Verträgen durch eine bemerkenswert kontinuierliche pro-europäische Grundhaltung aus, die trotz der Höhen und Tiefen des europäischen Integrationsprozesses nie wirklich in Frage gestellt worden ist. ${ }^{12} \mathrm{Zu}$ dieser Einschätzung kamen bereits Gary Marks und Carole J. Wilson, die auf Basis von Dokumentenanalysen und eines Experten-Surveys die Positionen nationaler Parteien zur europäischen Integration von 1984 bis 1996 vergleichend analysiert haben. ${ }^{13}$ Selbst die deutsche Wiedervereinigung, durch die die Bundesrepublik erheblich an Größe und damit an politischem Einfluss hinzugewonnen hat, führte nicht etwa, wie von der realistischen Schule prognostiziert worden war, zu einer Kehrtwende in den Bemühungen der deutschen Regierungen, den eigenen Staat aktiv in europäische Institutionen einzubinden, sondern unterstreicht die Normalität Deutschlands als integralem Bestandteil der Europäischen Union. ${ }^{14}$ Die für die

12 Demgegenüber ist die öffentliche Meinung zur europäischen Integration in Deutschland weniger pro-europäisch: Verglichen mit anderen EU-Staaten belegt die Bundesrepublik nur einen Mittelfeldplatz, wenn die Bürger gefragt werden, ob sie die Mitgliedschaft ihres Landes in der EU für eine gute Sache halten, und die Zustimmung nimmt seit den 1990er Jahren tendenziell ab; vgl. Guido Tiemann, Sozioökonomische Determinanten von Euroskeptizismus und Integrationsunterstützung, in: Martin Höpner / Armin Schäfer (Hrsg.), Die Politische Ökonomie der europäischen Integration, Frankfurt am Main 2008, S. 241 - 276. Zudem legen andere Studien die Vermutung nahe, die Mehrheit der Deutschen hätte nicht der Einführung des Euro oder der Osterweiterung zugestimmt, wenn Referenden durchgeführt worden wären; vgl. Jürgen Maier / Frank Brettschneider / Michaela Maier, Medienberichterstattung, Mediennutzung und die Bevölkerungseinstellungen zum Euro in Ost- und Westdeutschland, in: Frank Brettschneider / Jan van Deth / Edeltraut Roller (Hrsg.), Europäische Integration und öffentliche Meinung, Opladen 2003, S. 213 - 233; Stephen Wood, Germany and the Eastern Enlargement of the EU. Political Elites, Public Opinion and Democratic Processes, in: Journal of European Integration, 24. Jg. (2002), H. 1, S. $23-38$.

13 Gary Marks / Carole J. Wilson, The Past in the Present: A Cleavage Theory of Party Response to European Integration, in: British Journal of Political Science, 30. Jg. (2000), H. 3, S. 433 - 459.

14 Vgl. zu dieser These Adrian Hyde-Price / Charlie Jeffery, Germany in the European Union: Constructing Normality, in: Journal of Common Market Studies, 39. Jg. (2001), H. 4, S. 689 - 717; Jeffrey A. Anderson / John B. Goodman, Mars or Minerva? A United Germany in a Post Cold War Europe, in: Robert E. Keohane / Joseph S. Nye / Stanley Hoffmann (Hrsg.), After the Cold War: International Institutions and State Strategies in Europe 1989-1991, Cambridge 1993, S. 23 - 62. 
beiden deutschen Volksparteien ausschlaggebenden Beweggründe - einerseits die Idee, die Stellung Deutschlands im europäischen Machtgefüge durch den Transfer nationaler Souveränität auf die europäische Ebene bewusst zu schwächen und sich damit gewissermaßen selbst „zu zähmen“, andererseits das handfeste ökonomische Interesse an einem gemeinsamen Binnenmarkt, um die exportorientierte deutsche Wirtschaft zu stärken - haben trotz veränderter Rahmenbedingungen nichts an ihrer Gültigkeit verloren. ${ }^{15}$ Nachhaltige Spannungen und tiefgreifende Konflikte in den Einstellungen der beiden deutschen Volksparteien zur Europapolitik, so die vorherrschende Annahme, bestehen nach wie vor nicht und sind vor diesem Hintergrund auch in Zukunft nicht zu erwarten.

Diese Kernthese wird durch die jüngste Bundestagsdebatte zur Ratifikation des EU-Reformvertrages von Lissabon allerdings nur teilweise bestätigt: Grundsätzlich besteht sicher kein Zweifel daran, dass die pro-europäische Grundhaltung im deutschen Parteiensystem fortbesteht und ein radikaler Paradigmenwechsel auf der einen oder anderen Seite des politischen Spektrums aufgrund von Pfadabhängigkeiten selbst langfristig nicht abzusehen ist. Dementsprechend wurde in den Redebeiträgen zum Lissabon-Vertrag immer zunächst auf die historische Bedeutung der europäischen Einigung verwiesen und das Projekt Europa als Erfolgsgeschichte gewürdigt, die fortgesetzt werden sollte. Solange deutsche Regierungen und die Verwaltungen der Bundesministerien in den Verhandlungsprozess zur Vorbereitung von EU-Entscheidungen eingebunden bleiben und bereits früh in Absprache mit den Oppositionsparteien nationale Interessen einbringen können, wird der Bundestag weiterhin Gesetzentwürfe zur Änderung von EU-Grundlagenverträgen mit großen Mehrheiten ratifizieren. Keine der Parteien wird den Integrationsprozess europaweit zum Stillstand bringen wollen, und das Gesamtpaket wird in der Regel immer noch als bessere Option angesehen werden als ein Festhalten am Status quo, selbst wenn einzelne Abgeordnete bestimmte Teilaspekte dezidiert ablehnen. Ein Wandel in der Europapolitik der Volksparteien wird sich also nicht in sprunghaften Brüchen vollziehen, sondern in nuancierten Verschiebungen im Rahmen einer grundsätzlich positiven Europarhetorik.

Vor diesem pro-europäischen Hintergrund zeigt die Dokumentation der Redebeiträge der CDU/CSU- und SPD-Fraktion zum Vertrag von Lissabon im Bundestag einige richtungsweisende Wandlungstendenzen in der Europapolitik, insbesondere auf Seiten der Sozialdemokraten, die zu einer echten europapolitischen Kontroverse führen und das deutsche Parteiensystem langfristig in zwei Europalager spalten könnten: Nachdem in der Maastricht-Debatte bereits mehrere kleinere Konfliktherde zwischen der CDU/CSU-Fraktion und einigen SPD-Abgeordneten aufgetreten waren, vor allem in der Bewertung der Wirtschafts- und Währungsunion, die sich in der Nizza-Debatte dann nicht mehr zeigten, haben die Sozialdemokraten nun wieder zu einer moderaten Europaskepsis zurückgefunden, die vor allem das Fehlen einer „sozialen Dimension“ der europäischen Integration beklagt. Gleichzeitig verstärkte sich unter einigen Abgeordneten der CSU wieder die Forderung vor

15 Vgl. Peter Katzenstein, Gezähmte Macht: Deutschland in Europa, in: Michèle Knodt / Beate Kohler-Koch (Hrsg.), Deutschland zwischen Europäisierung und Selbstbehauptung, Frankfurt am Main / New York 2000, S. 57 - 84; Andrew Moravcsik, The Choice for Europe. Social Purpose and State Power from Messina to Maastricht, Ithaca 1998; siehe aktuell zur Positionierung der deutschen Bundesregierung zum EU-Verfassungsvertrag Stephanie Daimer / Thomas König, Germany. The Promoter of European Integration?, in: Thomas König / Simon Hug (Hrsg.), PolicyMaking Processes and the European Constitution. A Comparative Study of Member States and Accession Countries, London / New York 2006, S. 102 - 109. 
allem aufgrund von parlamentarischen Kontrollverlusten und verfassungsrechtlichen Bedenken, nicht immer mehr nationalstaatliche Souveränität an die Europäische Union zu übertragen und das Subsidiaritätsprinzip im europapolitischen Tagesgeschäft endlich praktisch wirksam werden zu lassen, also nur dann europaweite Regelungen zu erlassen, wenn Probleme nicht auf nationaler oder regionaler Ebene effektiver und sachgerechter gelöst werden können. Zwar wollen auch Merkel und Beckstein das Prinzip der sozialen Marktwirtschaft im Europa-Kontext erhalten; allerdings hatten sie bei der Ludwig-Erhard-Formel immer schon anderes im Sinn als die SPD, wenn sie von sozialer Gerechtigkeit sprach. ${ }^{16}$

Einer der Gründe für die Renaissance der Idee eines „sozialen Europas“ ist zweifellos der Aufstieg der Linkspartei, die der SPD permanent ihre vermeintlich unsoziale Politik vorwirft und durch ihre Wahlerfolge, vor allem in Ostdeutschland, zu einem ernstzunehmenden Gegner für die Sozialdemokraten avanciert ist. Zusätzlich zu diesem Druck vom linken Rand des politischen Spektrums haben die veränderten ökonomischen Rahmenbedingungen im europäischen Binnenmarkt durch die EU-Osterweiterung die Bedeutung der sozialen Dimension unterstrichen. Auch wenn erste Reformschritte in Deutschland unternommen wurden, um die lokalen Standortbedingungen dem verschärften Wettbewerb in der EU anzupassen, haben die meisten der mittel- und osteuropäischen Neumitglieder nach wie vor vergleichsweise niedrige Sozialstaatsniveaus, wenig regulierte Arbeitsmärkte und geringere Steuersätze für ausländische Unternehmen, so dass die Konkurrenz um kostengünstige Produktionsstandorte massiv zugenommen hat. Linksgerichtete Parteien sind mehr oder weniger gezwungen, sozialpolitische und arbeitsrechtliche Regulierungen auf EU-Ebene zu fordern, wenn sie zwar den Weggang von Industrieunternehmen verhindern, aber gleichzeitig die Errungenschaften des nationalen Sozialstaats retten wollen. Diese Situation kann schnell in eine Zwickmühle führen, da aufgrund der Interessen insbesondere der neuen Mitgliedstaaten, die überwiegend vom Status quo profitieren, die europaweite Einführung tiefgreifender Sozialstandards kaum zu erwarten ist. ${ }^{17}$ Insofern hat die Politisierung der europäischen Integration den Streit um wirtschaftspolitische Konzepte nun endgültig von der nationalen auf die EU-Ebene ausgeweitet. ${ }^{18}$

Vorausgesetzt, diese neuen Zukunftsideen zur Europapolitik sind nicht nur weitere Spielarten bedeutungsloser Europarhetorik, sondern sollen tatsächlich Eingang finden in europapolitische Sachentscheidungen, dann liegt hier der Nährboden nicht nur für die Wiederbelebung, sondern für die Intensivierung einer alten Konfliktlinie zwischen den beiden Volksparteien: Während die CDU/CSU-Fraktion die Aufgabe nationaler Souveränität zukünftig auf die Kernbereiche der Europäischen Union begrenzen will, insbesondere auf Regelungen zur Erleichterung freier Marktwirtschaft in einem europäischen Binnenmarkt, um nicht weiter die nationalen demokratischen Institutionen zu schwächen, fordert die

16 Vgl. Dieter Erb, 60 Jahre CDU - Ludwig Erhard und der Mythos Soziale Marktwirtschaft, in: Neue Gesellschaft Frankfurter Hefte, 52. Jg. (2005), H. 6, S. 42 - 46; Martin Nonhoff, Politischer Diskurs und Hegemonie: Das Projekt Soziale Marktwirtschaft, Bielefeld 2006.

17 Vgl. Fritz Scharpf, The European Social Model. Coping with the Challenges of Diversity, in: Journal of Common Market Studies, 40. Jg. (2002), H. 4, S. 645 - 670; Stephan Leibfried I Herbert Obinger, Nationale Sozialstaaten in der Europäischen Union: Zukünfte eines „sozialen Europas", in: Martin Höpner / Armin Schäfer (Hrsg.), a.a.O. (Fn. 12), S. 335 - 365.

18 Vgl. zu dieser These auch Liesbet Hooghe / Gary Marks, Die Entstehung eines politischen Gemeinwesens: Der Kampf um die europäische Integration, in: Martin Höpner / Armin Schäfer (Hrsg.), a.a.O. (Fn. 12), S. 159 - 195. 
SPD eine europäische Sozialpolitik und damit weitere Integrationsschritte in einem Politikfeld, das bisher immer noch weitgehend nationaler Regelungskompetenz unterliegt und dessen Vergemeinschaftung einen wesentlichen Bereich nationalstaatlicher Politik auf die europäische Ebene verlagern würde. Diese Zukunftsvisionen lassen sich offensichtlich nur schwer miteinander vereinbaren. Sollte die "Sozialstaatswerdung “ Europas ${ }^{19}$ an der CDU/ CSU-Fraktion (und den Regierungen anderer Mitgliedstaaten) scheitern, ist das Fortbestehen des Konsenses der beiden deutschen Volksparteien in europapolitischen Grundsatzfragen durchaus fraglich.

19 Wilhelm Knelangen, „Sozialstaatswerdung“ Europa? Integrationstheoretische Überlegungen zur Entwicklung der EU-Sozialpolitik, in: Alexandra Baum-Ceisig / Anne Faber (Hrsg.), Soziales Europa? Perspektiven des Wohlfahrtsstaates im Kontext von Europäisierung und Globalisierung, Wiesbaden 2005, S. $20-44$.

\title{
Das Integrationsverantwortungsgesetz - ein Kurzkommentar
}

\author{
Sven Hölscheidt, Steff Menzenbach und Birgit Schröder*
}

Das Integrationsverantwortungsgesetz (IntVG) gehört einer seltenen Gattung an, dem echten Parlamentsgesetz. ${ }^{1}$ Es ist das Kernstück des Gesetzes über die Ausweitung und Stärkung der Rechte des Bundestages und des Bundesrates in Angelegenheiten der Europäischen Union $^{2}$, das am 25. September 2009 in Kraft getreten ist. In nur drei Monaten hat der Bundestag dieses Begleitgesetz $z^{3}$ und vier weitere Gesetzentwürfe ${ }^{4}$ erarbeitet und insgesamt

* Die Autoren sind Mitarbeiter der Wissenschaftlichen Dienste des Deutschen Bundestags. Der Beitrag gibt ausschließlich ihre persönliche Auffassung wieder.

1 Vgl. Gunther Krichbaum, BT-Plenarprotokoll 16/233, S. 26350 (B). Über 80 Prozent der beschlossenen Gesetze sind Regierungsvorlagen; viele der als Bundestagsinitiativen verbuchten Entwürfe werden lediglich parallel zum Regierungsentwurf eingebracht; vgl. Deutscher Bundestag, Referat Parlamentsdokumentation, Statistik der Gesetzgebung - Überblick 16. Wahlperiode, Stand vom 5. November 2009, http://www.bundestag.de/dokumente/parlamentsdokumentation/ gesetzgebung.pdf (Abruf am 23. November 2009).

2 BGBl. I 2009, S. 3022.

3 BT-Drs. 16/13923.

4 BT-Drs. 16/13924; BT-Drs. 16/13925; BT-Drs. 16/13926; BT-Drs. 16/13928. Alle Entwürfe waren Gegenstand einer Gemeinsamen Öffentlichen Anhörung der Europaausschüsse von Bundestag und Bundesrat am 26. und 27. August 2009: Armin von Bogdandy (A-Drs. Nr. 16[21]914 [neu]); Christian Calliess (A-Drs. Nr. 16[21]912); Andreas Fisahn (keine schriftliche Stellungnahme); Christian Hillgruber (A-Drs. Nr. 16[21]904); Ingolf Pernice (A-Drs. Nr. 16[21]915); Franz C. Mayer (A-Drs. Nr. 16[21]916); Dietrich Murswiek (A-Drs. Nr. 16[21]910); Andreas Peter Maurer (A-Drs. Nr. 16[21]913); Adelheid Puttler (A-Drs. Nr. 16[21]905); Matthias Ruffert (ADrs. Nr. 16[21]908); Jürgen Schwarze (A-Drs. Nr. 16[21]909); Rudolf Streinz (A-Drs. Nr. 16[21]911). Ihre schriftlichen Stellungnahmen und das Wortprotokoll der Anhörung, Protokoll Nr. 90 des EU-Ausschusses, http://www.bundestag.de/bundestag/ausschuesse/a21/anhoerungen/90_sitzung/index.html (Abruf am 9. November 2009); Berichterstattung über die Anhörung: 